\title{
Strange stars and the cosmological constant problem
}

\author{
Cosimo Bamb:* \\ Istituto Nazionale di Fisica Nucleare, Sezione di Ferrara, I-44100 Ferrara, Italy \\ Dipartimento di Fisica, Università degli Studi di Ferrara, I-44100 Ferrara, Italy
}

(Dated: November 15, 2018)

\begin{abstract}
The cosmological constant problem represents an evident tension between our present description of gravity and particle physics. Many solutions have been proposed, but experimental tests are always difficult or impossible to perform and present phenomenological investigations focus only on possible relations with the dark energy, that is with the accelerating expansion rate of the contemporary universe. Here I suggest that strange stars, if they exist, could represent an interesting laboratory to investigate this puzzle, since their equilibrium configuration is partially determined by the QCD vacuum energy density.
\end{abstract}

\section{INTRODUCTION}

According to General Relativity, spacetime geometry is determined by the matter energymomentum tensor, so that somehow we could measure the value of vacuum energy, i.e. the energy of the ground state. On the other hand, neglecting General Relativity, only differences in energy are relevant. Even if we are not able to compute vacuum energy with any confidence, particle physics would suggest a huge value [1], in contrast with clear evidences, which require an almost flat spacetime around us. This is the well known cosmological constant problem [2] and represents an evident tension between our present description of gravity and particle physics. The problem is made more mysterious by observational data, which show an accelerating expansion rate of the universe and favor a tiny but non-zero vacuum energy density [3].

In this paper I focus the attention on strange stars [4, 5], where gravitational properties of QCD vacuum play an important role in the stellar equilibrium. Here hadrons have been converted into weakly interacting quarks and QCD vacuum energy density is different from the one in the confined phase. This makes strange stars an interesting laboratory to investigate the cosmological constant problem. If the true solution to this puzzle was the existence of some adjustment mechanism [6, 7], which compensates any effective cosmological constant in the Einstein field equation, it could also work inside the strange star, determining its equilibrium configuration: its effect could be roughly

\footnotetext{
${ }^{*}$ E-mail: bambi@fe.infn.it
} 
as the effective source of the gravitational field was the matter energy-momentum tensor without bare cosmological constants. Of course, the situation for quark stars and for the whole universe is not exactly the same: the formers are objects of size of about $10 \mathrm{~km}$ while the latter has practically an infinite radius. However, we can expect that the compensating mechanism works inside the star, whereas finite size effects are important only in a thin layer on the star surface. If this is the case, depends on the features of the adjustment mechanism, whose construction is not the purpose of the present work. The true solution to the problem may also be the existence of some other unknown reason, whose final result is basically the same, that is the effective cancellation of any cosmological constant in the gravitational field equation. Here I assume just that some mechanism exists and acts in most of the stellar interior and I study observational signatures which could distinguish this possibility from the standard case.

The content of the paper is as follows. In Section 2, I review particle physics estimates of the value of the cosmological constant. In Section 3, I discuss the special environment provided by strange stars and, in Section 4, the possible role of adjustment mechanisms for the star equilibrium configuration. In Section [5 and Section 6, there are the numerical results, respectively for the case without and with adjustment mechanism. In Section 7, I discuss the results and possible implications for future observations. In Section [8, there are summary and conclusion. Eqs. (18), (19), (20) and (21) are clarified in Appendix A, A short review on present possibilities and future prospects of measurement of mass and radius of compact stars is reported in Appendix B.

\section{EFFECTIVE COSMOLOGICAL CONSTANT FROM PARTICLE PHYSICS}

There exist apparently many sources capable of contributing to an effective cosmological constant $\Lambda_{e f f}$, so that an accidental and almost exact cancellation of all these pieces appears quite improbable.

First of all, the Einstein-Hilbert action with a bare cosmological constant term would represent the most general action (in four dimensions) of the gravitational sector satisfying "reasonable" requirements, leading to tensor field equation that contains up to second order derivatives of the metric. Of course, if $\Lambda \neq 0$ a matter free spacetime is not flat, but at present there are no theoretical reasons to believe that the latter must be Minkowskian.

At the classical level, we could expect a non-null energy density of the ground state for every 
field. For the sake of simplicity, let us consider a real scalar field $\phi$ with action

$$
S=\int d^{4} x \sqrt{-g}\left[\frac{1}{2} g^{\mu \nu} \partial_{\mu} \phi \partial_{\nu} \phi-V(\phi)\right]
$$

Its energy-momentum tensor is

$$
T_{\mu \nu}=\partial_{\mu} \phi \partial_{\nu} \phi-\frac{1}{2} g_{\mu \nu} g^{\kappa \lambda} \partial_{\kappa} \phi \partial_{\lambda} \phi+V(\phi) g_{\mu \nu}
$$

and in the state with lowest energy (if it exists) the kinetic energy is zero and $\phi$ is sitting at the minimum of the potential, so that Eq. (2) becomes

$$
T_{\mu \nu}=V\left(\phi_{\min }\right) g_{\mu \nu}
$$

Hence, we could naively expect a vacuum energy density of about $(100 \mathrm{GeV})^{4}$ in the context of the Standard Model of particle physics and a larger contribution, at the level of $M_{G U T}^{4} \sim\left(10^{16} \mathrm{GeV}\right)^{4}$, if we believe in GUTs. Moreover, it is common belief that some phase transitions took place in the early universe. This is quite intriguing, because it means that the effective cosmological constant changed several times in the history of the universe and that today is nearly zero. For example, at the electroweak phase transition the difference in the vacuum energy density between the symmetric and the broken phase was about $(100 \mathrm{GeV})^{4}$, while at the QCD phase transition about $(100 \mathrm{MeV})^{4}$. The usual and unsatisfactory assumption is that the minima of potentials in particle physics are exactly zero: this is not difficult to realize, for example taking $V(\phi)=\lambda\left(\phi^{2}-\phi_{\min }^{2}\right)^{2}$ in Eq. (11). In addition to this, since present astrophysical and cosmological observations suggest an accelerated phase of the present expansion rate of the universe [3], the introduction of a very light $(m<H)$ field, which is not yet at the minimum of its potential today, is often used to explain the data.

At the quantum level, a huge cosmological constant could arise from the zero point energy density. In flat spacetime, the energy density of the vacuum state $|0\rangle$ for a bosonic field of mass $m_{b}$ and $g_{b}$ internal states is

$$
\rho_{v a c}^{b}=\left\langle 0\left|T_{00}\right| 0\right\rangle=\frac{g_{b}}{4 \pi^{2}} \int_{0}^{M} \sqrt{k^{2}+m_{b}^{2}} k^{2} d k \sim \frac{g_{b}}{16 \pi^{2}} M^{4} .
$$

Here $M$ is the cut-off energy scale above which standard quantum field theory breaks down and is usually expected at the Planck scale, that is about $10^{19} \mathrm{GeV}$. On the other hand, because of anti-commutation relations, the energy density of the vacuum state for a fermionic field of mass $m_{f}$ and $g_{f}$ internal states is

$$
\rho_{v a c}^{f}=\left\langle 0\left|T_{00}\right| 0\right\rangle=-\frac{g_{f}}{4 \pi^{2}} \int_{0}^{M} \sqrt{k^{2}+m_{f}^{2}} k^{2} d k \sim-\frac{g_{f}}{16 \pi^{2}} M^{4} .
$$


As put forward for the first time by Zeldovich [8], if each fermionic degree of freedom had a bosonic counterpart with the same mass, the total zero point energy density would be zero. It is worth of noting that the idea was suggested before the advent of supersymmetry and for completely different reasons. However, even if supersymmetry is realized in nature, it must be broken at least at the $\mathrm{TeV}$ scale and the related total energy density should be about $(1 \mathrm{TeV})^{4}$.

\section{QUARK MATTER}

An interesting contribution to the cosmological constant can be expected from the non-trivial structure of the QCD vacuum. In fact, even if the vacuum contains no hadrons, it is not completely empty: quantum fluctuation of quark and gluon fields have non-vanishing average density (vacuum condensates). Since the existence and the value of these condensates influence hadron properties (for example, the quark condensate sets the pion mass), we can deduce them from particle physics experiments (see e.g. Ref. [9]). At a renormalization scale of $1 \mathrm{GeV}$, the value of the $u$ and $d$ quark condensates are

$$
\langle 0|\bar{u} u| 0\rangle_{Q=1 \mathrm{GeV}} \sim\langle 0|\bar{d} d| 0\rangle_{Q=1 \mathrm{GeV}} \sim-(240 \mathrm{MeV})^{3} .
$$

Since the energy-momentum tensor of a fermionic field $\psi$ contains the term $m \bar{\psi} \psi g_{\mu \nu}$, from Eq. (6) we should expect an effective cosmological constant

$$
\Lambda \sim m_{q}\langle 0|\bar{q} q| 0\rangle \sim-(100 \mathrm{MeV})^{4}
$$

Another contribution of the same order of magnitude should arise from the gluon condensate, whose estimate is

$$
\frac{\alpha_{s}}{\pi}\left\langle 0\left|G_{\mu \nu}^{a} G_{a}^{\mu \nu}\right| 0\right\rangle \sim(330 \mathrm{MeV})^{4}
$$

At high temperature and/or high baryon number density, ordinary hadron matter is expected to transform into deconfined quarks (quark-gluon plasma). These conditions could be reached for short time in heavy ion collisions and, for much longer time and larger amount of matter, in the core of neutron stars. There exists also the possibility that strange quark matter, that is quark matter made of $u, d$ and $s$ quarks, could be absolutely stable [5, 10]: since quarks are fermions, introducing a third flavor there are new low energy available states, reducing the total energy of the system (for an introduction, see e.g. [11] and references therein). If true, strange stars made of strange quark matter could exist and be the ground state of neutron stars. The peculiar feature 
in the deconfined phase is that chiral symmetry is restored and the quark vacuum condensate, which represents the order parameter of chiral symmetry breaking, goes to zero: $\langle 0|\bar{q} q| 0\rangle \rightarrow 0$. The gluon vacuum condensate also changes value and at the transition is probably about one half of the estimate of Eq. (8). Because of the different value of QCD vacuum energy density in the two phases, we can expect that large amounts of quark matter, so large that gravity is non-negligible, can give us informations about the cosmological constant problem.

Quark matter inside strange stars can be described as a Fermi gas at zero temperature of massless quarks living in a space with energy density $B$ [11]. In this simple picture, the quark matter energy-momentum tensor can be written as

$$
T_{\mu \nu}=T_{\mu \nu}^{\text {Fermi gas }}+T_{\mu \nu}^{\text {vacuum }}
$$

where $T_{\mu \nu}^{\text {Fermi gas }}$ describes the Fermi gas and $T_{\mu \nu}^{\text {vacuum }}=B g_{\mu \nu} . B$ is a constant representing the difference in energy density between the QCD vacua in the deconfined and confined phase. This is essentially the MIT bag model [12], even if the vacuum energy density $B$ is not exactly the bag constant we can deduce from hadron spectroscopy: the common value $B=60 \mathrm{MeV} \mathrm{fm}^{-3}$ is probably not good for the description of quark matter and a higher value, for example $B \approx 100$ $\mathrm{MeV} \mathrm{fm}^{-3}$ (as suggested by the computation of hadronic structure functions [13]), appears more appropriate. The energy-momentum tensor of Eq. (9) can be written as the one of a perfect fluid with energy density $\rho$ and pressure $P$ given by

$$
\begin{aligned}
\rho & =A n^{4 / 3}+B, \\
P & =\frac{A}{3} n^{4 / 3}-B .
\end{aligned}
$$

Here $n$ is the baryon number density and $A$ a constant. For $N_{f}$ massless flavors and to first order in the QCD coupling $\alpha_{s}$, the constant $A$ is [14]

$$
A=\frac{9}{4}\left(\frac{3 \pi^{2}}{N_{f}}\right)^{1 / 3}\left(1+\frac{8 \alpha_{s}}{3 \pi}\right)
$$

In the following I take $N_{f}=3$ and $\alpha_{s}=0.5$, so that $A=6.87$.

\section{ADJUSTMENT MECHANISM}

Since there exist many different sources capable of contributing to an effective cosmological constant and we have to expect that some of them changed during the history of the universe, one of the most promising solutions is represented by the so-called adjustment mechanisms (some 
examples can be found in [6, 7]), because they do not require any fine-tuning and are not sensitive to the particular nature of the source. Moreover, these models can also explain the present accelerating expansion rate of the universe, since typically they are unable to erase completely a bare cosmological constant; it is also intriguing that the first model [6] was proposed long before universe acceleration was discovered.

The basic idea is that cosmological constant-like terms stimulate the formation of the condensate of some field, whose energy density compensates the energy density of the source: the final result is that gravity, just like all the other non-gravitational phenomena, becomes sensitive only to differences in energy. Here I do not want to consider a particular model, whose peculiar features are usually relevant only in the time interval between the appearance of a new effective cosmological constant, for example after the phase transition, and its compensation, when the equilibrium has been restored by the adjustment mechanism. In addition to this, I would like to include possible other mechanisms, whose final result is the same. For what follows, it is sufficient to assume that the presence in the matter energy-momentum tensor of the term $\Lambda_{e f f} g_{\mu \nu}$ stimulates the appearance of a counterterm $\sim-\Lambda_{e f f} g_{\mu \nu}$ on the right hand side of the Einstein field equation, so that cosmological constant-like terms do not play any role in spacetime geometry. Since the energy-momentum tensor of quark matter contains the term $B g_{\mu \nu}$, inside strange stars the adjustment mechanism should introduce a compensating term $\sim-B g_{\mu \nu}$, which cancels (or reduces considerably) the former. What happens on the star surface depends on the particular model; however, if the transition region is thin, it is not relevant for the star equilibrium.

This very simple rearragement of Einstein field equation is usually a reasonable approximation after equilibrium restoration, that is long after the effective cosmological constant was compensated 6, 7]. During the backreaction process, additional model-dependent and non-negligible terms of the compensating field have to appear in the Einstein equation. However, even if we do not know the exact adjustment mechanism, we can expect that the compensation process is very rapid and does not affect the stellar equilibrium "at later time". This statement is based on the following consideration. In the early universe, when the temperature dropped down to about 100 $\mathrm{MeV}$, which corresponds to an age of the universe of $10^{-4} \mathrm{~s}$, quark-gluon plasma converted into hadrons and, since QCD vacuum energy changed, a new cosmological constant appeared. In models with adjustment mechanisms, the compensating field had to neutralize this cosmological constant in a very short time, because the Big Bang Nucleosynthesis is capable of explaining primordial abundances only with a standard expansion rate of the universe [15]: it means that 1 second after QCD transition, the compensation was essentially completed. As for the onset of the phase 
transition, which is expected to be of first order inside strange stars, it depends only on QCD parameters. Non-standard physics can enter after this event, but in this case it can only favor the conversion from hadron to quark matter, because in the standard theory $T_{\mu \nu}^{\mathrm{vacuum}}$ offers resistance to the gravitational collapse (see next sections), preventing a higher baryon density and the related more favorable condition for the transition to the deconfined phase.

\section{STANDARD PREDICTIONS}

Writing the Einstein field equations for a perfect fluid with spherical distribution, we get the Tolman-Oppenheimer-Volkoff equations [16]

$$
\begin{aligned}
\frac{d P}{d r} & =-\frac{G_{N}[\rho(r)+P(r)]\left[M_{G}(r)+4 \pi r^{3} P(r)\right]}{r\left[r-2 G_{N} M_{G}(r)\right]}, \\
\frac{d M_{G}}{d r} & =4 \pi r^{2} \rho(r) .
\end{aligned}
$$

Here $r$ is the radial coordinate, $P(r)$ the pressure, $\rho(r)$ the energy density and $M_{G}(r)$ the gravitational mass inside $r$. Even if some exact solutions of these equations are known, realistic equations of state require to be solved numerically. As for strange stars, in the standard theory (i.e. without adjustment mechanism) the energy-momentum tensor which appears on the right hand side of the Einstein equations is the one in (9) and the corresponding energy density and pressure are given in Eqs. (10) and (11). Thus, Eqs. (13) and (14) with the equation of state $P=(\rho-4 B) / 3$ can be solved numerically with initial conditions

$$
\begin{aligned}
P(0) & =\frac{A}{3} n_{C}^{4 / 3}-B, \\
M_{G}(0) & =0,
\end{aligned}
$$

where $n_{C}$ is the baryon number density at the center of the compact object and represents the only free parameter. The integration ends at the star surface, placed at $r=R$ and defined by

$$
P(R)=\frac{A}{3} n^{4 / 3}(R)-B=0 .
$$

The results are reported in Figure 1 for $B=80 \mathrm{MeV} \mathrm{fm}^{-3}$ (blue dashed curves). For different values of the vacuum energy density $B$, the maximum mass and the corresponding equilibrium radius scale with $B$ as (see Appendix $\mathrm{A}$ )

$$
\begin{aligned}
M_{\text {max }} & =1.63\left(\frac{80 \mathrm{MeV} \mathrm{fm}^{-3}}{B}\right)^{1 / 2} M_{\odot}, \\
R\left(M_{\max }\right) & =9.15\left(\frac{80 \mathrm{MeV} \mathrm{fm}^{-3}}{B}\right)^{1 / 2} \mathrm{~km} .
\end{aligned}
$$




\section{STRANGE STARS IN PRESENCE OF SOME COMPENSATING FIELD}

The presence of some compensating field introduces into the Einstein field equation a counterterm which essentially cancels $T_{\mu \nu}^{\mathrm{vacuum}}$. In this case, hydrostatic equilibrium is always given by Eqs. (13) and (14), but now we have to set $B$ to zero. On the other hand, $B$ remains in Eq. (17) for the definition of the star radius, because non-gravitational physics is essentially unchanged: the compensating field interacts only gravitationally. In particular, a number of possible phenomena, such as color superconductivity, Cooper pair condensation an so on, are not directly affected by the presence of the adjustment mechanism; at most indirectly, because of the modification of the energy density profile. In other words, the pressure in Eq. (17) is not the total gravitational pressure appearing in the Einstein equation and source of the gravitational field, but only the pressure produced by quark matter, and the condition (17) basically means that there is a threshold baryon number density for the existence of the deconfined phase (in fact, it implies $\rho(R)=4 B$ which, for typical values of $B$, is somewhat more than the energy density in ordinary nuclear matter). The definition of star surface is a subtle point mostly because in our model independent picture we do not know exactly what happens at the boundary between different QCD vacua, but conserving the condition (17) is certainly the most reasonable possibility.

Moreover, I would like to remark that here we are considering the star equilibrium "at late time". During the phase transition from hadron to quark matter and the subsequent backreaction, which takes back the effective cosmological constants to zero (or to a tiny value), the picture is more complicated and strongly model-dependent. In particular, we should know energy exchange processes between standard matter and the compensating field, the related response time and so on.

The results for $B=80 \mathrm{MeV} \mathrm{fm}^{-3}$ are in Figure 1 (solid red curves). In the table below, there are maximum mass and related radius and star baryonic charge for other value of the vacuum energy density $B$. 

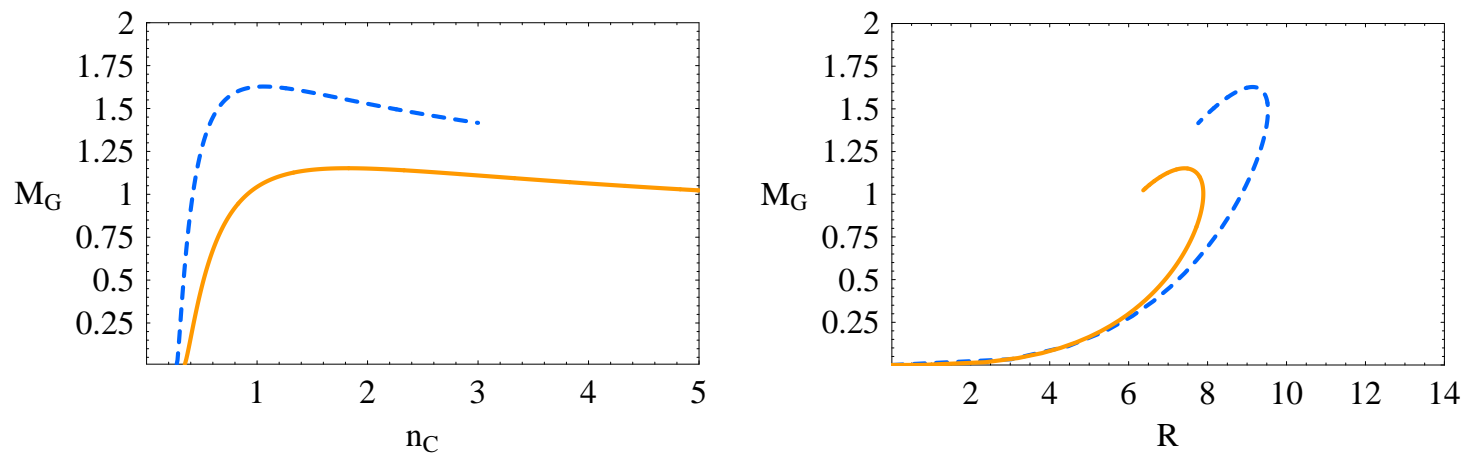

FIG. 1: Left panel: gravitational mass $M_{G}$ in Solar mass unit as a function of the central baryon number density $n_{C}$ in $\mathrm{fm}^{-3}$. Right panel: gravitational mass $M_{G}$ in Solar mass unit as a function of the equilibrium radius $R$ in $\mathrm{km}$. Results for $B=80 \mathrm{MeV} \mathrm{fm}^{-3}$; blue dashed curves for the standard case and red solid curves in presence of some compensating field.

\begin{tabular}{||c|c|c|c||}
\hline \hline$B /\left(\mathrm{MeV} \mathrm{fm}^{-3}\right)$ & $M_{\max } / M_{\odot}$ & $R\left(M_{\max }\right) / \mathrm{km}$ & $N_{B}\left(M_{\max }\right) / 10^{57}$ \\
\hline \hline 60 & 1.33 & 8.57 & 1.66 \\
\hline 80 & 1.15 & 7.42 & 1.34 \\
\hline 100 & 1.03 & 6.63 & 1.13 \\
\hline 120 & 0.94 & 6.06 & 0.99 \\
\hline 140 & 0.87 & 5.60 & 0.88 \\
\hline 160 & 0.81 & 5.24 & 0.80 \\
\hline \hline
\end{tabular}

As in the standard case, $M_{\max }$ and $R\left(M_{\max }\right)$ scale with $B$ as ${ }^{1}$

$$
\begin{aligned}
M_{\max } & =1.15\left(\frac{80 \mathrm{MeV} \mathrm{fm}^{-3}}{B}\right)^{1 / 2} M_{\odot}, \\
R\left(M_{\max }\right) & =7.42\left(\frac{80 \mathrm{MeV} \mathrm{fm}^{-3}}{B}\right)^{1 / 2} \mathrm{~km} .
\end{aligned}
$$

\section{DISCUSSION}

Cosmological constant-like terms in the matter energy-momentum tensor produce a sort of "anti-gravity" (if $\Lambda_{e f f}>0$ ), in the sense that they offer resistance to the gravitational collapse of the star: this is the same effect of a positive cosmological constant for the expansion of the universe. The term in Eq. (13) responsible for this effective gravitational repulsion is $M_{G}+4 \pi r^{3} P$,

\footnotetext{
${ }^{1}$ The $B$-dependence of $M_{\max }$ and $R\left(M_{\max }\right)$ could appear strange in this case, but the definition of the stellar surface (17) preserves the $B$ scale law in the hydrostatic equations. For more details, see Appendix A
} 
where the presence of vacuum energy introduces an extra contribution equal to $-8 / 3 \pi r^{3} B$. This implies that the compensating mechanism makes strange star maximum mass $M_{\max }$ decrease, that strange stars are more compact and, for a fixed gravitational mass, have a smaller radius $R$ (Figure 1, right panel). One can also easily see that the presence/absence of $B$ in Eqs. (13) and (14) gives an order one effect on $M_{\max }$ : the repulsive vacuum energy density force to the gravitational mass ratio is (for more details see Ref. [17])

$$
\beta(r)=\frac{\frac{8}{3} \pi r^{3} B}{M_{G}(r)}
$$

and, for $r=8 \mathrm{~km}, B=100 \mathrm{MeV} \mathrm{fm}^{-3}$ and $M_{G}=M_{\odot}$, we get $\beta=0.4$.

Another important quantity is the star baryonic charge, given by

$$
N_{B}=\int_{0}^{R} \frac{n(r)}{\left(1-\frac{2 G_{N} M(r)}{r}\right)^{1 / 2}} 4 \pi r^{2} d r .
$$

For a fixed gravitational mass, $N_{B}$ is slightly higher for the non-standard case, see Figure 2, This is obvious, because the compensating field erases the contribution from vacuum energy density to $M_{G}$ (or equivalently it give a negative contribution to $M_{G}$ ), which in the standard case is

$$
M_{G}=\int_{0}^{R}\left(A n^{4 / 3}+B\right) 4 \pi r^{2} d r .
$$

Even if quite rough, the description of quark matter I have used is capable of showing the relevant differences between the two cases (some considerations on more realistic models are reported below). In particular, the most important feature is represented by the quite low maximum mass in presence of the compensating mechanism. A possible future observation of a quark star with mass of 1.4 $M_{\odot}$ could certainly disfavor the existence of mechanisms capable of erasing $T_{\mu \nu}^{\mathrm{vacuum}}$-like terms in the Einstein field equation. On the other hand, if some compensating field exists and works inside strange stars, these very compact objects would be very rare, even for the most favorable QCD parameters: in fact, stellar evolution favors compact stars with a mass of about $1.4 M_{\odot}$, whose baryon number is $N_{B} \sim 1.7 \cdot 10^{57}$ : these are probably values too high for a strange star of Section 6 for any reasonable value of $B$. Since stars with mass larger than $M_{\max }$ are unstable, we can expect that they have to collapse into black holes just after or during the transition from the confined to the deconfined phase. Finally, identification of strange stars with unexpected low masses would represent a sign of the compensation of the QCD vacuum energy density ${ }^{2}$.

\footnotetext{
${ }^{2}$ For a possible strange star candidate, see [18] and references therein. However, for this object no measurements of mass and radius are available at present.
} 
As for possible modifications of this picture from more sophisticated descriptions of quark matter, we can easily see that they do not change significantly these conclusions. For example, here we have considered three massless fermions: even if it is a good approximation for $u$ and $d$ quarks, because they are much lighter than the Fermi energy, a non-zero $m_{s}$, at the level of 100 $\mathrm{MeV}$, may be important. This is indeed true, but $m_{s} \neq 0$ makes $M_{\text {max }}$ decrease (see e.g. Ref. [19]) and the effects of the compensating mechanism more evident. A more subtle point is represented by effects of stellar rotation. Slow rotation [20] makes $M_{\max }$ increase, but no more than about 10 - 20\%, and this increment can be compensated (at least partially) by a non-zero strange quark mass. On the other hand, fast rotation is much more complicated to take into account, even with standard physics [21]. More study would be desirable, but radical changes cannot be expected.

Then, we can also consider the possibility that strange matter is not absolutely stable. This happens for higher $B$ and heavier $m_{s}$ : for example, for $m_{s} \approx 0 \mathrm{MeV}(150 \mathrm{MeV})$ strange quark matter is stable at zero pressure if $B \lesssim 100 \mathrm{MeV} \mathrm{fm}^{-3}\left(80 \mathrm{MeV} \mathrm{fm}^{-3}\right)$ (see e.g. Ref. [19]). However, it is quite reasonable that quark matter can exist at higher densities, in the center of compact stars. In this case we talk of "hybrid stars", stars with a core of quark matter and an outer part of hadron matter. Of course, if the quark matter core is large enough, the previous conclusions do not require any change. On the other hand, compensating mechanism effects become less and less important in the stellar equilibrium for a smaller and smaller quark matter core. What really happens in nature depends only on QCD physics and at present we can not make solid predictions. Additional ambiguity is introduced because we do not know the lifetime of the possible metastable state, that is how long time is needed for the transition from the confined to the deconfined state to take place (it may be practically instantaneous as well as be much longer than the age of the universe). However, all these uncertainties are due to our poor knowledge of QCD and do not depend directly on the compensating mechanism. Thanks to the efforts of many people in the topic, at both theoretical and experimental level, in a near future the standard picture could be much clearer.

Finally, I would like to note that present considerations cannot be applied to single hadrons: according to the MIT bag model, hadrons are basically described as bubbles of quark matter and one may naively think of observing some effect of the compensating mechanism in their gravitational properties. However, this cannot happen, otherwise we should immediately reject this kind of solutions, because we should expect a number of phenomena, such as violations of the so-called Equivalence Principle [22]. For example, the Newton gravitational constant measured in Cavendishlike experiments should differ at the level of $25 \%$ (i.e. the contribution of QCD vacuum energy 


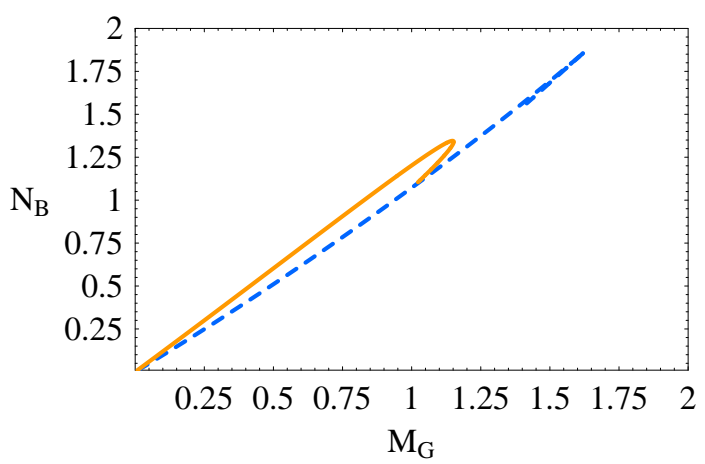

FIG. 2: Baryon number of the star $N_{B}$ in $10^{57}$ unit as a function of the gravitational mass $M_{G}$ in Solar mass unit. Results for $B=80 \mathrm{MeV} \mathrm{fm}^{-3}$; blue dashed curves for the standard case and red solid curves in presence of some compensating field.

density in the inertial mass of hadrons in the MIT bag model) from the one we commonly use in cosmology in the radiation dominated universe, where the expansion is not determined by hadrons. But there are at least two reasons since this is not a problem. First, hadrons are objects much smaller than strange stars, so finite size effects can be very important and the compensating mechanism can be unable to work. Second, hadrons are non-perturbative objects and it is quite reasonable that their properties cannot be always deduced by a model where they are small bubbles with two or three free quarks and/or antiquarks inside.

\section{CONCLUSION}

Even if according to General Relativity spacetime geometry is sensitive to vacuum energy, particle physics estimates and observational evidences could suggest it is not true. This is the well known cosmological constant problem. Many solutions have been proposed, but at present no one appears satisfactory; a non-trivial difficulty towards the solution of the puzzle is certainly represented by the essential absence of observational or experimental tests (with the exclusion of present universe acceleration, which may or may not be related to the problem), which could give us informations and hints on the approach to follow.

In this paper I have focused the attention on strange stars, where QCD vacuum energy density of the deconfined phase is expected to contribute in the determination of the star equilibrium. If strange stars were allowed by QCD, they would represent an extraordinary laboratory for fundamental physics and, among other things, could answer interesting questions about the cosmological constant problem. 
If cosmological constant-like terms had to be compensated by some unknown adjustment mechanism (or if there exists some other reason so that gravity is insensitive to vacuum energy), strange stars would have a quite low maximum mass and this would represent a clear signature to distinguish this possibility from the one we can expect just combining General Relativity with particle physics. Strange stars, if they exist, can play an important role in the solution to the cosmological constant problem.

\section{Acknowledgments}

I wish to thank Alexander Dolgov, Alessandro Drago and Irene Parenti for useful discussions and suggestions.

\section{APPENDIX A: $B$ SCALING}

In the standard case, with the equation of state $P=(\rho-4 B) / 3$, Eqs. (13) and (14) can be written as the differential equation

$$
\frac{d \rho}{d r}=-\frac{16 \pi G_{N}}{3} \frac{[\rho(r)-B]\left[3 \int_{0}^{r} \rho(x) x^{2} d x+r^{3} \rho(r)-4 r^{3} B\right]}{r^{2}-8 \pi G_{N} r \int_{0}^{r} \rho(x) x^{2} d x}
$$

with initial condition $\rho(0)=\rho_{C}$ and definition of star surface $\rho(R)=4 B$. It is easy to see that this equation is invariant under the transformation

$$
\begin{aligned}
B & \rightarrow B_{*}, \\
\rho(r) & \rightarrow \rho_{*}\left(r_{*}\right)=\rho(r) \frac{B_{*}}{B}, \\
r & \rightarrow r_{*}=r\left(\frac{B}{B_{*}}\right)^{1 / 2} .
\end{aligned}
$$

Hence, the star radius $R$ and the gravitational mass $M_{G}=\int 4 \pi x^{2} \rho d x$ scale as $B^{-1 / 2}$. This explains Eqs. (18) and (19).

However, if we put $B=0$ in Eq. (A1), the new hydrostatic equation with the same boundary conditions $\rho(0)=\rho_{C}$ and $\rho(R)=4 B$ continues to be invariant under the transformation (A2), so that $M_{\max }$ and $R\left(M_{\max }\right)$ are always proportional to $B^{-1 / 2}$. This is exactly the results we find from numerical integration, see Eqs. (20) and (21). 


\section{APPENDIX B: MEASUREMENT OF MASS AND RADIUS}

Just like for ordinary neutron stars, a simultaneous measurement of mass and radius of an intermediate mass strange star would provide interesting informations and, in our case, could help to discriminate among the two picture and teach us something about the cosmological constant problem. However, the observational determination of the two quantities is not so easy for this kind of objects (neutron stars and possible strange stars).

As for the mass, very accurate measurements, even with relative errors of about $0.1 \%$, can be obtained if the compact star is a pulsar in a binary system (see e.g. Ref. [23]). Masses can also be determined if the compact star is in an X-ray binary and is accreting matter from a companion, but the accuracy is much lower, no better than $10 \%$.

On the other hand, radius measurements are much more difficult and confused. If we know the distance of the compact star and we measure its flux and luminosity on the Earth, we can deduce the radiation radius, defined as

$$
R_{\infty}=\frac{R}{1-\frac{2 G_{N} M_{G}}{R}}
$$

and, from observation of spectra lines, we would be able to determine both $R$ and $M_{G}$ separately. However, at present the exact identification of lines is quite problematic [24]. Good measurements of $R_{\infty}$ could be also available in a near future from compact stars in binaries in globular clusters, thanks to quiescent X-ray bursts, if the measurements of the distance to their globular clusters will be improved [25].

Other future possibilities of determination of mass and radius could involve neutrino flux from proto-compact stars (see [26] and references therein) and gravitational wave observations [27].

[1] Ya.B. Zeldovich, JETP Lett. 6, 316 (1967); Pisma Zh. Eksp. Teor. Fiz. 6, 883 (1967).

[2] A.D. Dolgov, in Proc. of the XXIV Rencontre de Moriond, p. 227 (ed. J. Adouse and J. Tran Thanh Van, Les Arcs, France, 1989);

S. Weinberg, Rev. Mod. Phys. 61, 1 (1989).

[3] S. Perlmutter et al. (Supernova Cosmology Project Collaboration), Astrophys. J. 517, 565 (1999), arXiv:astro-ph/9812133

A.G. Riess et al. (Supernova Search Team Collaboration), Astron. J. 116, 1009 (1998), arXiv:astro-ph/9805201 
D.N. Spergel et al. (WMAP Collaboration), Astrophys. J. Suppl. 148, 175 (2003), arXiv:astro-ph/0302209

[4] N. Itoh, Prog. Theor. Phys. 44, 291 (1970).

[5] E. Witten, Phys. Rev. D 30, 272 (1984).

[6] A.D. Dolgov, in The Very Early Universe, p.449 (ed. G.W. Gibbons, S.W. Hawking and S.T.C. Siklos, Cambridge University Press, Cambridge, UK, 1983).

[7] L.H. Ford, Phys. Rev. D 35, 2339 (1987);

A.D. Dolgov, Phys. Rev. D 55, 5881 (1997), arXiv:astro-ph/9608175

S. Mukohyama and L. Randall, Phys. Rev. Lett. 92, 211302 (2004), arXiv:hep-th/0306108;

S. Alexander and D. Vaid, arXiv:hep-th/0702064.

[8] Ya.B. Zeldovich, Sov. Phys. Usp. 11, 381 (1968); Usp. Fiz. Nauk 95, 209 (1968).

[9] T. Schäfer and E.V. Shuryak, Rev. Mod. Phys. 70, 323 (1998).

[10] A.R. Bodmer, Phys. Rev. D 4, 1601 (1971).

[11] J. Madsen, Lect. Notes Phys. 516, 162 (1999), arXiv:astro-ph/9809032.

[12] A. Chodos, R.L. Jaffe, K. Johnson, C.B. Thorn and V.F. Weisskopf, Phys. Rev. D 9, 3471 (1974);

T. DeGrand, R.L. Jaffe, K. Johnson and J. Kiskis, Phys. Rev. D 12, 2060 (1975).

[13] F.M. Steffens, H. Holtmann and A.W. Thomas, Phys. Lett. B 358, 139 (1995), arXiv:hep-ph/9508398.

[14] G. Chapline and M. Nauenberg, Nature (London) 264, 235 (1976).

[15] C. Bambi, M. Giannotti and F.L. Villante, Phys. Rev. D 71, 123524 (2005), arXiv:astro-ph/0503502.

[16] R.C. Tolman, Phys. Rev. 55, 364 (1939);

J.R. Oppenheimer and G.M. Volkoff, Phys. Rev. 55, 374 (1939).

[17] C. Bambi, Phys. Rev. D 75, 083003 (2007), arXiv:astro-ph/0703645.

[18] A. Drago, G. Pagliara and I. Parenti, arXiv:0704.1510 [astro-ph].

[19] I. Parenti, Deconfinamento dei quark nelle stelle di neutroni, Degree Thesis, Pisa University (2003).

[20] M. Colpi and J.C. Miller, Astrophys. J. 388, 513 (1992).

[21] E. Gourgoulhon et al., Astron. Astrophys. 349, 851 (1999), arXiv:astro-ph/9907225.

[22] C.M. Will, Living Rev. Rel. 9, 3 (2006), arXiv:gr-qc/0510072.

[23] N. Manchester and J.H. Taylor, Pulsar (W.H. Freeman and Company, San Francisco, USA, 1977).

[24] C.J. Hailey and K. Mori, Astrophys. J. 578, L133 (2002), arXiv:astro-ph/0207590.

[25] C.O. Heinke, J.E. Grindlay, D.A. Lloyd and P.D. Edmonds, Astrophys. J. 588, 452 (2003), arXiv:astro-ph/0301235,

R.E. Rutledge, L. Bildsten, E.F. Brown, G.G. Pavlov and V.E. Zavlin, Astrophys. J. 578, 405 (2002), arXiv:astro-ph/0105405.

[26] M. Prakash, J.M. Lattimer, R.F. Sawyer and R.R. Volkas, Ann. Rev. Nucl. Part. Sci. 51, 295 (2001), arXiv:astro-ph/0103095.

[27] M. Prakash and J.M. Lattimer, J. Phys. G 30, S451 (2004), arXiv:astro-ph/0305306. 